\title{
Freezing of soft spheres: A critical test for weighted-density-functional theories
}

\author{
Brian B. Laird and D.M. Kroll \\ Institut für Festkörperforschung, Forschungszentrum Jülich G.m.b.H., \\ Postfach 1913, D-5170 Jülich 1, Federal Republic of Germany
}

(Received May 22 1990)

\begin{abstract}
We study the freezing properties of systems with inverse-power and Yukawa interactions (soft spheres), using recently developed weighted-density-functional theories. We find that the modified weighted-density-functional approximation (MWDA) of Denton and Ashcroft yields results for the liquid to face-centered-cubic (fcc) structure transition that represent a significant improvement over those of earlier "second-order" density-functional freezing theories; however, this theory, like the earlier ones, fails to predict any liquid to body-centered-cubic (bcc) transition, even under conditions where the computer simulations indicate that this should be the equilibrium solid structure. In addition, we show that both the modified effective-liquid approximation (MELA) of Baus [J. Phys. Condens. Matter 1, 3131 (1989)] and the generalized effective-liquid approximation of Lutsko and Baus [Phys. Rev. Lett. 64, 761 (1990)], while giving excellent results for the freezing of hard spheres, fail completely to predict freezing into either fcc or bcc solid phases for soft inverse-power potentials. We also give an alternate derivation of the MWDA that makes clearer its connection to earlier theories.
\end{abstract}

\section{INTRODUCTION}

Over the past decade, the use of classical densityfunctional theory (DFT) has become increasingly popular in the study of solid-liquid phase transitions. ${ }^{1,2}$ The popularity of these methods is, at least in part, due to their success at providing an accurate description of the fluid to close-packed solid transition of a system of hard spheres. ${ }^{3-11}$ Similar encouraging results have also been obtained for the freezing of a Lennard-Jones liquid into a face-centered-cubic (fcc) solid. ${ }^{4,12,13}$ On the basis of these results, it seems that density-functional theory works well-at least for systems of monatomic particles with "hard" interactions (i.e., those with steep repulsive parts) for which the equilibruim solid phase is close packed. A natural question now arises: Does the success of DFT in the case of "hard" potentials extend as well to systems of particles with "softer" interactions, where the thermodynamically stable solid phase is not necessarily close packed? This question was answered, in part, by Barrat et al. ${ }^{14}$ in a systematic study of the freezing properties of repulsive inverse-power potentials:

$$
v(r)=\epsilon\left(\frac{\sigma}{r}\right)^{n} .
$$

For a class of density functionals in which the solid free-energy functional is approximated by a second-order functional Taylor expansion about a homogeneous reference phase, Barrat et al. showed that the densities at which the liquid and fcc solid co-exist are consistently overestimated; the disagreement with the computersimulation data ${ }^{15-18}$ increases dramatically as softer (smaller $n$ ) potentials are considered (from about $10 \%$ in the $n=12$ case to over $100 \%$ for $n=4$ ). More damaging is the failure of these "second-order" theories to predict, for any value of $n$, a stable body-centered-cubic (bcc) solid phase (or even a metastable one, for that matter). This result is in direct contradiction to the computersimulation findings that the equilibrium crystal phase at freezing should have a (non-close-packed) bcc structure for $n \leq 6 .{ }^{19}$ Therefore, the truncation of the functional expansion at second order does not result in a suitable description of the solid phase for these soft-sphere systems, and higher-order contributions must somehow be taken into account. Unfortunately, a straightforward extention of these density-functional perturbation methods to include the higher-order effects is difficult since the third- or higher-order direct correlation functions of the homogeneous liquid phase, which would be the coefficients of such an expansion, are, in general, unknown. (Some attempts at including approximate expressions for the three-body direct-correlation functions in the freezing calculation have, however, met with some qualitative success. $^{20,21}$ )

Recently, several versions of DFT, $8,10,11,22$ belonging to the class of so-called weighted-density-functional theories (WDFT), have been proposed. By weighting the local density with a suitably chosen weighting function, they achieve an approximate resummation of the higherorder terms in the solid free-energy functional, while still requiring knowledge of only the two-particle direct correlations in the homogeneous liquid. All of these new formulations give good freezing results for the hard-sphere (HS) system. ${ }^{8,10,11}$

In contrast to the earlier theories, these new methods also yield well-defined free-energy minima for the bcc hard-sphere solid, as long as the shear-mode fluctuations, to which the bcc HS solid is known to be unstable (see Sec. III), are a priori excluded from the calculation. The resulting bcc free energies compare well with the results 
of Monte Carlo calculations performed within a similarly restricted phase space. ${ }^{23}$

In this work, we undertake a systematic study of the freezing properties of two types of soft spheres using weighted-density-functional methods in order to determine whether or not the implicit addition of approximate higher-order terms corrects the qualitative deficiencies of the "second-order" theories for these systems. The first potential we consider is the previously mentioned inversepower interaction, defined in Eq. (1). The second involves spheres interacting via a repulsive Yukawa potential,

$$
v(r)=\epsilon \frac{e^{-\kappa r}}{r},
$$

which is a very good model of the interactions in monodisperse colloids. ${ }^{24,25}$ The advantage of using these two systems for the present study is that the range of interaction of both potentials can be tuned over a wide range through the variation of a single parameter ( $n$ for inverse powers and $\kappa$ for the Yukawa spheres). Also, good computer-simulation phase-transition data are available for both interaction types.

By comparing the weighted-density-functional results for these systems with both the "second-order" DFT results and the computer-simulation findings, we will, hopefully, obtain a clearer picture of the current state of the density-functional theory of freezing. In this sense our current work complements and extends that of Barrat et al. ${ }^{14}$ In the following section, we briefly summarize the various forms of density-functional theory considered in this work, with special emphasis on the weighted-density formalisms. A new derivation of the modified weighteddensity approximation of Denton and Ashocroft ${ }^{10}$ is also presented that makes clearer its connection to earlier theories. Sections III and IV contain the results of our weighted-density-functional study of the freezing of the inverse-power and Yukawa systems. These results are compared with those of earlier theories and with the computer-simulation findings. In Sec. V, we summarize our results and make some concluding remarks.

\section{SUMMARY OF DENSITY-FUNCTIONAL FORMALISM}

In the canonical ensemble, a density-functional theory is a procedure for determining the Helmholtz free energy $F$ associated with a given spatially dependent singleparticle density $\rho(\mathbf{r})$; that is, $F$ is determined as a functional of $\rho(\mathbf{r})$. The equilibrium free energy and microscopic density can then be found by minimizing this functional, $F[\rho]$, over the space of single-particle densitiessubject to the constraint that the volume and total number of particles remains fixed. For a detailed description of basic classical density-functional theory and its mathematical justifications, see the review by Evans. ${ }^{26}$

The functional $F[\rho]$ can be written as the sum of an ideal part $F_{\mathrm{id}}[\rho]$ and an excess part $F_{\mathrm{ex}}[\rho]$ due to the interparticle interactions:

$$
F[\rho]=F_{\text {id }}[\rho]+F_{\text {ex }}[\rho] .
$$

The ideal part is known exactly, and for a monatomic system is given by

$$
F_{\mathrm{id}}[\rho]=\int d \mathbf{r} \rho(\mathbf{r})\left\{\ln \left[\Lambda^{3} \rho(\mathbf{r})\right]-1\right\},
$$

where $\Lambda$ is the thermal wavelength. The excess part is, in general, unknown; therefore, the central task of a densityfunctional theory is to provide a suitable approximation scheme for this quantity.

Most density-functional theories begin by defining the $n$-body direct-correlation functions $c^{(n)}\left(\mathbf{r}_{1}, \ldots, \mathbf{r}_{n} ;[\rho]\right)$ in terms of the functional derivatives of $F_{\mathrm{ex}}[\rho]$ :

$$
c^{(n)}\left(\mathbf{r}_{1}, \ldots, \mathbf{r}_{n} ;[\rho]\right)=-\frac{\delta^{(n)} \beta F_{\mathrm{ex}}[\rho]}{\delta \rho\left(\mathbf{r}_{1}\right) \cdots \rho\left(\mathbf{r}_{n}\right)} .
$$

For arbitrary $\rho(\mathbf{r})$, these correlation functions, like $F[\rho]$ itself, are unknown, except in the homogeneous density (liquid) limit, where, due to the advances in liquid-state theory over the past three decades, they can be determined for $n \leq 2$. Density-functional theories of freezing exploit this knowledge of the liquid correlation functions to try to obtain approximations for those in an inhomogeneous phase.

The first and simplest DFT of freezing is due to Ramakrishnan and Yussouff. ${ }^{27}$ This theory was later reformulated in the language of classical density functionals by Haymet and Oxtoby ${ }^{28}$ (HO). In this theory, the free energy of the inhomogeneous phase (here, the solid) is expanded in a functional Taylor expansion about a reference liquid density. This expansion is subsequently truncated at second order to yield

$$
\begin{aligned}
& \beta F_{\mathrm{ex}}[\rho]= \beta F_{\mathrm{ex}}(\hat{\rho})-c_{l}^{(1)}(\hat{\rho}) \int d \mathbf{r}_{1}\left[\rho\left(\mathbf{r}_{1}\right)-\hat{\rho}\right] \\
&-\iint d \mathbf{r}_{1} d \mathbf{r}_{2} c_{l}^{(2)}\left(\left|\mathbf{r}_{1}-\mathbf{r}_{2}\right| ; \hat{\rho}\right) \\
& \times\left[\rho\left(\mathbf{r}_{1}\right)-\hat{\rho}\right]\left[\rho\left(\mathbf{r}_{2}\right)-\hat{\rho}\right]+\cdots
\end{aligned}
$$

In the Haymet-Oxtoby formulation, the reference density is chosen to be that of the equilibrium liquid, and for that reason, the functional that is minimized is actually the grand-canonical-potential functional obtained from $F[\rho]$ by Legendre transform:

$$
\Omega[\rho]=F[\rho]-\mu \int d \mathbf{r} \rho(\mathbf{r}) .
$$

A modification of this "second-order" approach was later developed by Baus and Colot and is called the effective-liquid approximation (ELA). ${ }^{7}$ The free-energy functional they consider is of the same form as the HO functional [Eq. (6)] with the reference density chosen to equal the bulk solid density; however, the function $c^{(2)}\left(\left|\mathbf{r}_{1}-\mathbf{r}_{2}\right| ; \hat{\rho}\right)$ is not evaluated at the reference density, but at some "effective" density chosen such that the first peak of the Fourier transform of $c^{(2)}$ coincides with the first nonzero reciprocal-lattice vector of the solid. 
In the hope of correcting the deficiencies of the second-order DFT's, a new approach was initiated by Tarazona. ${ }^{29}$ This weighted-density-functional method is a modification of the usual local-density approximation (LDA) for inhomogeneous systems. In the LDA, the freeenergy density at a point $\mathbf{r}$ in a system with inhomogeneous single-particle density $\rho(\mathbf{r})$ is given by the free energy of a homogeneous system, evaluated at the value of the single-particle density at point $\mathbf{r}$. However, for very strongly inhomogeneous systems such as a solid, the LDA breaks down. To remedy this, the local density is averaged over a small region using a weighting function $w\left(\left|\mathbf{r}_{1}-\mathbf{r}_{2}\right| ; \hat{\rho}\right)$ to create a coarse-grained or "weighted" density $\hat{\rho}(\mathbf{r})$ :

$$
\hat{\rho}\left(\mathbf{r}_{1}\right)=\int d \mathbf{r}_{2} \rho\left(\mathbf{r}_{2}\right) w\left(\left|\mathbf{r}_{1}-\mathbf{r}_{2}\right| ; \hat{\rho}\left(\mathbf{r}_{1}\right)\right) .
$$

The local-density approximation is then applied to this weighted density; that is, the free-energy functional is given by

$$
\beta F[\rho]=\int d \mathbf{r} \beta f_{0}(\hat{\rho}(\mathbf{r})) \rho(\mathbf{r}),
$$

where $f_{0}(\rho)$ is the excess Helmholtz free energy per particle of a homogeneous system of density $\rho$. The task of a successful weighted-density-functional theory is to choose a weighting function that leads to a good description of the structure and thermodynamics of the inhomogeneous phase.

These weighted-density-functional theories can be further divided into two classes. The first and computationally more involved class assumes a spatially inhomogeneous weighted density. The weighted-denstity approximation (WDA) of Curtin and Ashcroft ${ }^{8}$ is of this type. The second, simpler class assumes instead a constant weighted density. This approach was first taken by Denton and Ashcroft in their modified weighteddensity-functional approximation (MWDA); ${ }^{10}$ except for the form of the weighted density, this theory makes the same fundamental assumptions as the earlier and more complicated WDA. The very recent modified effectiveliquid approximation (MELA) of $\mathrm{Baus}^{22}$ and generalized effective-liquid approximation (GELA) of Lutsko and Baus ${ }^{11}$ are of such a form that they can be formulated using either a constant or a spatially dependent weighted density with only minor changes in the resulting equations.

In the weighted-density approximation of Curtin and Ashcroft, ${ }^{8}$ the weighting function $w\left(\left|\mathbf{r}_{1}-\mathbf{r}_{2}\right| ; \hat{\rho}\right)$ is chosen such that both the free energy and the two-particle direct-correlation function $c^{(2)}$ [as defined in Eq. (5)] are exactly reproduced in the limit of a homogeneous density. The first of these two requirements is guaranteed if the weighting function is assumed to be normalized to unity:

$$
\int d \mathbf{r}_{2} w\left(\left|\mathbf{r}_{1}-\mathbf{r}_{2}\right| ; \hat{\rho}\right) \equiv 1
$$

The second requirement determines the form of $w$ : functionally differentiating $\beta F_{\text {ex }}[\rho]$ [as defined by Eqs. (8)(10)] twice, taking the homogeneous limit $\rho(\mathbf{r}) \rightarrow \rho$, and setting the result equal to the negative of the homogeneous two-particle direct-correlation function $c^{(2)}$ at density $\rho$, as prescribed by Eq. (2) (with $n=2$ ), results in an integro-differential equation for the weighting function in terms of $c^{(2)}$. This equation is most easily represented and solved in Fourier space:

$$
\begin{aligned}
-\beta^{-1} c^{(2)}(k ; \rho)= & 2 f_{0}^{\prime}(\rho) w(k ; \rho)+\rho f_{0}^{\prime \prime}(\rho) w(k ; \rho)^{2} \\
& +2 \rho f_{0}^{\prime}(\rho) w^{\prime}(k ; \rho) w(k ; \rho),
\end{aligned}
$$

where a prime denotes differentiation with respect to density. After solving this equation for the weighting function, Eq. (8) can then be solved self-consistently (both the left and right sides of the equation depend upon the weighted density) to determine the weighted density for a given real density. The self-consistency requirement assures that the sum rule

$$
\frac{d}{d \rho} c^{(n)}\left(\mathbf{r}_{1}, \ldots, \mathbf{r}_{n}\right)=\int d \mathbf{r}_{n+1} c^{(n+1)}\left(\mathbf{r}_{1}, \ldots, \mathbf{r}_{n+1}\right),
$$

which follows from Eq. (5), is satisfied by the WDA functional in the homogeneous density limit. This sum rule ensures that the functional includes to infinite order all parts of the higher-order correlation functions that are fixed by the thermodynamics. This weighted density is then used to calculate the excess free energy via Eq. (9).

As one can see, the implementation of the WDA is rather involved. In the simpler MWDA of Denton and Ashcroft, ${ }^{10}$ the free energy of the inhomogeneous phase is given by the homogeneous free energy evalulated at a spatially independent weighted density:

$$
\beta F_{\mathrm{ex}}[\rho]=N \beta f_{0}(\hat{\rho}),
$$

where the weighted density is defined by

$$
\hat{\rho}=\frac{1}{N} \int d \mathbf{r}_{1} \rho\left(\mathbf{r}_{1}\right) \int d \mathbf{r}_{2} \rho\left(\mathbf{r}_{2}\right) w\left(\left|\mathbf{r}_{1}-\mathbf{r}_{2}\right| ; \hat{\rho}\right) \text {. }
$$

The weighting function $w$ is determined from the same two requirements used in the WDA. The advantage of the MWDA is that the resulting equation for the weighting function is not a complicated differential equation as is the WDA, but is easily solved algebraically to give

$$
\begin{aligned}
& w\left(\left|\mathbf{r}_{1}-\mathbf{r}_{2}\right| ; \hat{\rho}\right)=-\frac{1}{2 \beta f_{0}^{\prime}(\hat{\rho})}\left(c^{(2)}\left(\left|\mathbf{r}_{1}-\mathbf{r}_{2}\right| ; \hat{\rho}\right)\right. \\
& \left.+\frac{\hat{\rho} \beta f_{0}^{\prime \prime}}{V}\right)
\end{aligned}
$$

Since the MWDA satisfies the same set of sum rules as the WDA and therefore includes the same subset of "exact" higher-order terms, the MWDA should be regarded as occupying essentially the same level of approximation as the WDA for the class of problems considered here.

For the hard-sphere freezing transition, the predictions of the MWDA are nearly identical to those of the WDA, ${ }^{10}$ and, for the softer potentials considered in this work, pre- 
liminary calculations showed that there is little quantitative difference between the freezing results of the two approximations. One reason for this is that the weighting functions for the two methods are nearly identical in $k$ space for $k \sigma$ greater than a critical $k$ that is smaller than the typical first nonzero reciprocal-lattice vector in a crystal; therefore, as far as the freezing calculations are concerned, the weighting functions are nearly the same. The only major difference between the two formulations involves the evaluation of the free energy [Eq. (9) versus Eq. (13)]. Because of this similarity, the more easily calculable MWDA results will be taken in this paper as representative of both theories.

More recently, ${ }^{11}$ Lutsko and Baus proposed a WDFT of a different type. This method, the generalized effective-liquid approximation, is based on the following exact expression for the free-energy functional:

$$
\beta F_{\mathrm{ex}}[\rho]=-\int d \mathbf{r}_{1} \int d \mathbf{r}_{2} \int_{0}^{1} d \lambda \int_{0}^{\lambda} d \lambda^{\prime} \rho\left(\mathbf{r}_{1}\right) \rho\left(\mathbf{r}_{2}\right) c^{(2)}\left(\mathbf{r}_{1}, \mathbf{r}_{2} ;\left[\lambda^{\prime} \rho\right]\right),
$$

which follows by functionally intergrating the definition of the direct-correlation functions [Eq. (5)] with $n=2$. Assuming the correlation functions in an inhomogeneous system are well described by those of a homogeneous one evaluated at some reference density, Lutsko and Baus approximate Eq. (16) (which is exact) by

$$
\beta F_{\mathrm{ex}}[\rho]=-\int d \mathbf{r}_{1} \int d \mathbf{r}_{2} \int_{0}^{1} d \lambda \int_{0}^{\lambda} d \lambda^{\prime} \rho\left(\mathbf{r}_{1}\right) \rho\left(\mathbf{r}_{2}\right) c^{(2)}\left(\left|\mathbf{r}_{1}-\mathbf{r}_{2}\right| ; \hat{\rho}\left[\lambda^{\prime} \rho\right]\right)
$$

where $c^{(2)}(r ; \hat{\rho})$ is now taken to be the two-body directcorrelation function for a homogeneous system with density $\hat{\rho}$. The form of this functional is such that Eq. (5) is satisfied for all $n$ for all densities - not just in the homogeneous limit. A consequence of this is that the sum rules for higher-order direct-correlation functions [Eq. (12)] are automatically satisfied.

The functional $\hat{\rho}[\rho]$ is determined by requiring that the same reference liquid that is used in Eq. (17) to describe the correlation functions also describes the thermodynamics; that is,

$$
F_{\mathrm{ex}}[\rho]=N f_{0}(\hat{\rho}[\rho]) \text {. }
$$

Equations (17) and (18), then, uniquely define the density $\hat{\rho}$ for a given $\rho(\mathbf{r})$. It can be easily seen that this procedure is equivalent to using Eqs. (13) and (14) to define the functional, with the weighting function given by

$$
w\left(\left|\mathbf{r}_{1}-\mathbf{r}_{2}\right| ; \hat{\rho}\right)=-\frac{\hat{\rho} \int_{0}^{1} d \lambda \int_{0}^{\lambda} d \lambda^{\prime} c^{(2)}\left(\left|\mathbf{r}_{1}-\mathbf{r}_{2}\right| ; \hat{\rho}\left[\lambda^{\prime} \rho\right]\right)}{\beta f_{0}(\hat{\rho})}
$$

Note that this weighting function is normalized only in the homogeneous limit, which is all that is physically required.

The modified effective-liquid approximation of Baus consists of approximating the functional $\hat{\rho}[\lambda \rho]$ in Eqs. (17) and (19) by $\lambda \hat{\rho}[\rho]$. The MELA is computationally much simpler than the GELA, but has the disadvantage that it does not yield the correct $c^{(2)}$ in the homogeneous limit. It is interesting to note that the GELA and MELA can be formulated using a spatially dependent weighted density as in the WDA; the form of the weighting function does not change in this case. ${ }^{11}$

One drawback to the above formulation of the WDA and MWDA is that the existence of a weighting function is assumed a priori. It is possible, however, to derive both of these functionals by expanding the exact functional about a reference liquid and then choosing the reference density in a particularly optimal fashion. A consequence of this choice is that the resulting expression for the free energy of the inhomogeneous phase has the form of a weighted-density functional.

To derive the Denton-Ashcroft MWDA we start by expressing the exact functional as a functional integral over $c^{(1)}$ :

$$
\beta F_{e x}[\rho]=-\int d \mathbf{r} \int_{0}^{1} d \lambda \rho(\mathbf{r}) c^{(1)}(\mathbf{r} ;[\lambda \rho]) .
$$

This equation is simply the integral form of the definition of the single-particle direct-correlation function $c^{(1)}[\mathrm{Eq}$. (5) with $n=1]$. Similarly, one can expand $c^{(1)}(\mathbf{r} ;[\rho])$ about a homogeneous reference density $\hat{\rho}$ :

$$
\begin{aligned}
c^{(1)}\left(\mathbf{r}_{1} ;[\rho]\right)= & c^{(1)}(\hat{\rho}) \\
& +\int d \mathbf{r}_{2} \int_{0}^{1} d \lambda^{\prime} c^{(2)}\left(\mathbf{r}_{1}, \mathbf{r}_{2} ;\left[\rho_{\lambda^{\prime}}\right]\right)\left[\rho\left(\mathbf{r}_{2}\right)-\hat{\rho}\right]
\end{aligned}
$$

where $c^{(2)}\left(\mathbf{r}_{1}, \mathbf{r}_{2} ;[\rho]\right)$ is the two-particle direct-correlation functional as defined by Eq. (5) $(n=2)$ and the integration path is parametrized as follows:

$$
\rho_{\lambda}(\mathbf{r})=\hat{\rho}+\lambda[\rho(\mathbf{r})-\hat{\rho}] .
$$

Substituting this exact expression for $c^{(1)}$ into the above expression for $\beta F_{\mathrm{ex}}[\rho][\mathrm{Eq} .(20)]$ gives

$$
\begin{aligned}
\beta F_{\mathrm{ex}}[\rho]= & N \beta f_{0}(\hat{\rho}) \\
& -\int d \mathbf{r}_{1} \int d \mathbf{r}_{2} \rho\left(\mathbf{r}_{1}\right) \mathcal{C}\left(\mathbf{r}_{1}, \mathbf{r}_{2} ;[\rho]\right)\left[\rho\left(\mathbf{r}_{2}\right)-\hat{\rho}\right]
\end{aligned}
$$


where $f_{0}(\rho)$ is as defined previously and

$$
\left.\mathcal{C}\left(\mathbf{r}_{1}, \mathbf{r}_{2} ; \rho\right]\right) \equiv \int_{0}^{1} d \lambda \lambda \int_{0}^{1} d \lambda^{\prime} c^{(2)}\left(\mathbf{r}_{1}, \mathbf{r}_{2} ;\left[\lambda \rho_{\lambda^{\prime}}\right]\right)
$$

Since $\mathcal{C}$ is, for the most part, unknown, we approximate it by an as yet unspecified homogeneous two-point function $\mathcal{G}$ :

$$
\mathcal{C}\left(\mathbf{r}_{1}, \mathbf{r}_{2} ;[\rho]\right) \approx \mathcal{G}\left(\left|\mathbf{r}_{1}-\mathbf{r}_{2}\right| ; \hat{\rho}\right) .
$$

This approximation is due to Groot and van der Eerden ${ }^{30}$ who used it in their modification of the weighted-density-functional theory of Meister and Kroll. ${ }^{31}$

As in the GELA, we choose the reference density so that both the correlation functions and the free energy of the solid are described by the same reference liquid; that is, we require Eq. (13) to be valid ${ }^{32}$ This condition implies that the second term on the right-hand side of Eq. (23) is zero, which yields

$$
\hat{\rho}=\frac{1}{N} \int d \mathbf{r}_{1} \rho\left(\mathbf{r}_{1}\right) \int d \mathbf{r}_{2} \rho\left(\mathbf{r}_{2}\right) \frac{\mathcal{G}\left(\left|\mathbf{r}_{1}-\mathbf{r}_{2}\right| ; \hat{\rho}\right)}{\mathcal{G}(k=0 ; \hat{\rho})} .
$$

Finally, the function $\mathcal{G}$ is determined from the condition that the resulting free-energy functional yield the correct $c^{(2)}$ in the homogeneous density limit. Together with the compressibility equation,

$$
c^{(2)}(k=0 ; \hat{\rho})=2 \beta f_{0}^{\prime}(\hat{\rho})+\rho \beta f_{0}^{\prime \prime}(\hat{\rho}),
$$

this requirement gives

$$
\mathcal{G}\left(\left|\mathbf{r}_{1}-\mathbf{r}_{2}\right| ; \hat{\rho}\right)=c^{(2)}\left(\left|\mathbf{r}_{1}-\mathbf{r}_{2}\right| ; \hat{\rho}\right)+\frac{\hat{\rho} \beta f_{0}^{\prime \prime}(\hat{\rho})}{V} .
$$

A quick inspection shows that this procedure [defined by Eq. (13), (26), and (28)] is exactly the MWDA [defined by Eqs. (13)-(15)] if the correspondence, $\mathcal{G} / \mathcal{G}(k=0) \rightarrow w$, is made. A similar derivation of the Curtin and Ashcroft WDA requires only trivial modifications to the above procedure-details can be found in Ref. 33 .

Unless otherwise stated, all calculations reported in this paper were performed using a Gaussian parametrization for the solid single-particle density:

$$
\rho(\mathbf{r})=\left(\frac{\pi}{\alpha}\right)^{3 / 2} \sum_{\left\{\mathbf{R}_{i}\right\}} \exp \left(-\alpha\left|\mathbf{r}-\mathbf{R}_{i}\right|^{2}\right),
$$

where $\alpha$ is a measure of the width of the Gaussian peaks and $\left\{\mathbf{R}_{i}\right\}$ represents the set of real-space lattice vectors for the particular solid structure under consideration (for example, fcc or bcc). This parametrization simplifies the calculation considerably, because only a one-dimensional minimization (in $\alpha$ ) is required and many parts of the calculation can be done analytically; for example, the ideal part of the free energy [Eq. (4)] becomes simply

$$
\beta F_{\mathrm{id}}(\alpha)=\frac{3}{2} \ln \left(\frac{\alpha}{\pi}\right)+3 \ln (\Lambda)-\frac{5}{2} .
$$

The validity of this approximation is discussed in detail in Ref. 4.

\section{RESULTS FOR INVERSE-POWER POTENTIALS}

The inverse-power potential was defined in Eq. (1). This potential has two properties that make it useful as a test case for theories of freezing. First, the phase diagram is, for a given value of $n$, one dimensional. This is in contrast to the usual two-dimensional nature of phase diagrams for monatomic systems. This property, which greatly simplifies the calculations, follows from the fact that the length and energy scales ( $\sigma$ and $\epsilon$, respectively) of the potential are not independent. The potential depends on these parameters only through the combination $\epsilon \sigma^{n}$. Thus, all thermodynamic properties derivable from the configurational part of the free energy (this includes the melting and freezing points) are solely determined by the value of the combination density-temperature parameter

$$
\gamma_{n}=\rho \sigma^{3}\left(\frac{k T}{\epsilon}\right)^{-3 / n} .
$$

The second important property of this potential series is that, by varying the parameter $n$, one can compare the freezing properties of systems with widely varying ranges of interaction. The range of the inverse-power potential varies from the extremely short-ranged hardsphere interaction $(n=\infty)$ to the very long-ranged onecomponent-plasma potential $(n=1)$.

One additional advantage of using this potential series to test the predictions of freezing theories is the fact that both the phase transition properties and the equations of state have been studied in detail by computer simulation for $n=\infty$ (HS), ${ }^{34} 12,{ }^{15,16} 9,6$, and $4 .{ }^{17-19}$ These simulations supply an absolute standard to which a freezing theory can be compared.

An important result of these simulation studies concerns the equilibrium solid structure at freezing. For $n$ greater than about 7 , the liquid freezes into a fcc crystal sructure. Below this value of $n$, the bcc structure becomes the equilibrium freezing solid structure. In the region where the fcc phase is thermodynamically stable, the bcc phase is not only thermodynamically unstable, but is also mechanically unstable to shear. It is interesting to note that, for $n \geq 5$ (integer $n$ ), a zero-temperature lattice sum shows that fcc is the lower energy structure; ${ }^{19}$ therefore, for $5 \leq n \leq 6$, the thermodynamic stability of the bcc phase at temperatures near the melting point is of entropic origin. Lattice dynamics calculations show that the lowering of the bcc free energy (relative to fcc) is due to the existence of low-frequency shear modes. ${ }^{19,35}$ The correct description of this fcc to bcc transition would be a significant achievement for any freezing theory.

In this paper we study the freezing properties of this inverse power series for $n=12,6$, and 4 using the weighteddensity-functional theories MWDA, GELA, and MELA. 
The input liquid-structure data is calculated using the modified hypernetted-chain (MHNC) integral-equation method. ${ }^{35-38}$ For a description of the specific version of the technique we employ, see the article by Rosenfeld. ${ }^{39}$ The integral equation was solved numerically using the method of Labík, Malijevský, and Vońka. ${ }^{40}$ This integralequation method gives results that are in excellent agreement with computer-simulation data. As an example of the thermodynamic accuracy, Fig. 1 shows the liquid excess Helmoltz free energy per particle as a function of bulk number density for the inverse-sixth-power system as calculated using the MHNC and by integrating computer-simulation data. ${ }^{17}$ The agreement is excellent. Figure 2 shows the structure factor, $S(k)$, for the same system at freezing $\left(\gamma_{6}=2.18\right)$-also from both MHNC and computer simulation. Here, as well, the agreement is good.

Our results for the freezing of inverse-power liquids ( $n=12,6$, and 4 ) into an fcc solid are summarized in Table I. For comparison, the results of previous DFT calculations (including those for hard spheres) are also included. From this table, one sees that the MWDA does a better job than the "second-order" theories (HO and ELA) in predicting the fcc-liquid transition point. As with the "second-order" results, the predicted values of the freezing and melting points (calculated by Maxwell construction from the liquid and solid Helmholtz freeenergy curves) become much worse in comparison to the simulations as $n$ decreases. However, if one looks at the relative difference between the total free energies as predicted by the MWDA and those calculated from the computer-simulation data, one sees that this quantity actually decreases slightly as $n$ decreases. The values of the

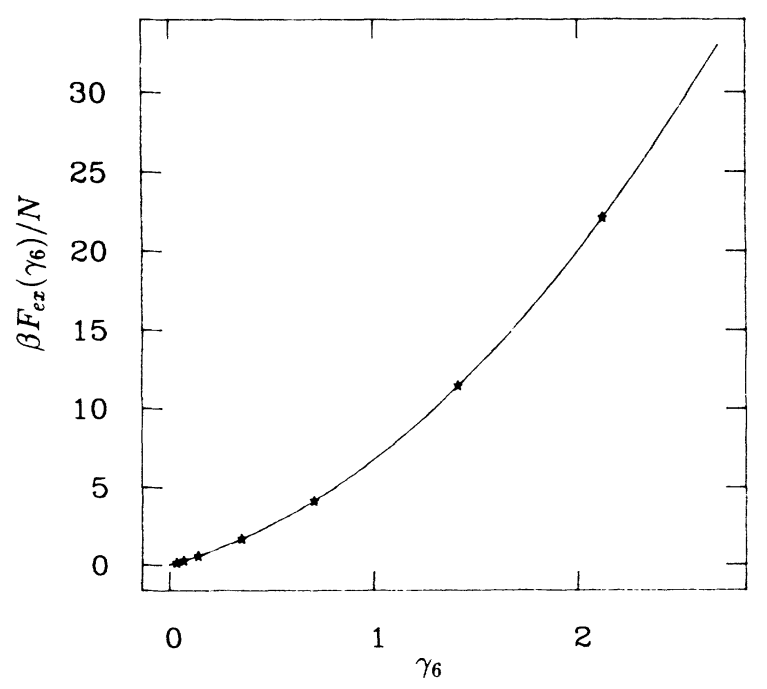

FIG. 1. Dimensionless excess Helmholtz free energy per particle for the inverse-sixth-power fluid as a function of $\gamma_{6}$. The solid line was calculated using the MHNC integralequation method and the stars represent computer-simulation data (Ref. 16).

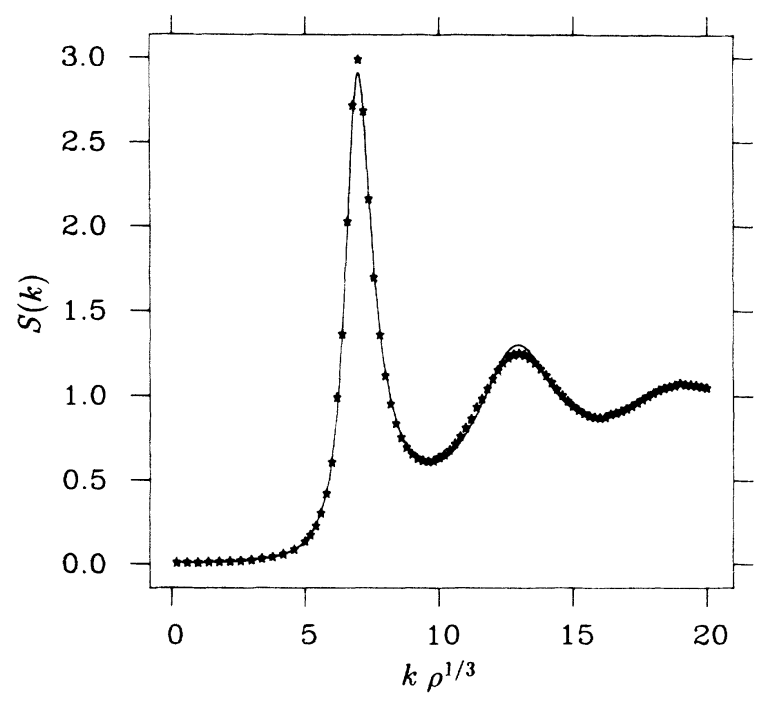

FIG. 2. Structure factor for an inverse-sixth-power fluid at the freezing point $\left(\gamma_{6}=2.18\right)$. The solid line is the MHNC integral-equation result and the stars are Monte-Carlo simulation data (Ref. 18).

MWDA fcc free energy for $n=6$ are compared in Table II with those of the computer simulations at various values of the density (the total free energies for the liquid, both from the MHNC and simulation data are also included). The decrease in the accuracy of the transitionpoint prediction is due, for the most part, to the fact that, as $n$ decreases, the angle at which the liquid and solid free-energy curves meet becomes smaller; therefore, for the more long-range potentials, a small error in the solid free-energy curve will lead to a much larger error in the point at which the two curves cross. The small crossing angle also accounts for the fact that the fractional density change on freezing $\Delta \rho / \rho_{s}$ decreases with decreasing $n$.

The MWDA does not do significantly better than the second-order theories in the determination of the Lindemann ratio $L$, defined as the ratio of the root-meansquared displacement of a particle about its lattice site to the ideal nearest-neighbor distance. (Note that the phenomenological Lindemann rule that, for a given system, $L$ should remain constant along the liquid-solid coexistence line, is exactly satisfied for potentials of the inverse-power type as a consequence of the scaling properties mentioned above.) As in the earlier theories, the predicted Lindemann ratios are about a factor of 2 below the simulation values. This discrepancy is, at least partially, due to the overestimation of the transition density, since the width of the solid density peaks decreases rapidly with increasing solid density.

The MWDA fails to find nontrivial $(\alpha \neq 0)$ minima in the bcc solid free energy. This lack of bcc minima persists even when possible anisotropic and anharmonic solid single-particle densities are taken into account through the addition of higher-order cubic harmonics to the argu- 
TABLE I. Comparison of fcc freezing results for inverse-power potentials.

\begin{tabular}{|c|c|c|c|c|c|}
\hline$n$ & Method & $\gamma_{f}$ & $\gamma_{s}$ & $\Delta \rho / \rho_{s}$ & $L$ \\
\hline \multirow[t]{6}{*}{$\infty$ (hard spheres) } & MWDA (Ref. 10) & 0.910 & 1.036 & 0.13 & 0.097 \\
\hline & GELA (Ref. 11) & 0.945 & 1.041 & 0.10 & 0.095 \\
\hline & MELA (Ref. 11) & 0.970 & 1.070 & 0.084 & 0.099 \\
\hline & ELA (Ref. 7) & 0.99 & 1.08 & 0.09 & 0.07 \\
\hline & HO (Refs. 4 and 14) & 0.967 & 1.147 & 0.18 & 0.06 \\
\hline & Simulation (Ref. 34) & 0.943 & 1.041 & 0.104 & 0.126 \\
\hline \multirow[t]{6}{*}{12} & MWDA (this work) & 1.194 & 1.252 & 0.046 & 0.096 \\
\hline & GELA (this work) & \multirow{2}{*}{\multicolumn{4}{|c|}{$\begin{array}{l}\text { no minima } \\
\text { no minima }\end{array}$}} \\
\hline & MELA (this work) & & & & \\
\hline & ELA (Ref. 14) & 1.305 & 1.380 & 0.06 & 0.07 \\
\hline & HO (Ref. 14) & 1.28 & 1.37 & 0.07 & 0.07 \\
\hline & Simulation (Ref. 16) & 1.15 & 1.19 & 0.035 & 0.15 \\
\hline \multirow[t]{6}{*}{6} & MWDA (this work) & 2.666 & 2.720 & 0.020 & 0.074 \\
\hline & GELA (this work) & \multirow{2}{*}{\multicolumn{4}{|c|}{$\begin{array}{l}\text { no minima } \\
\text { no minima }\end{array}$}} \\
\hline & MELA (this work) & & & & \\
\hline & ELA (Ref. 14) & 3.33 & 3.39 & 0.02 & 0.07 \\
\hline & HO (Ref. 14) & 3.43 & 3.52 & 0.026 & 0.07 \\
\hline & Simulation (Ref. 16) & 2.18 & 2.21 & 0.013 & 0.17 \\
\hline \multirow[t]{6}{*}{4} & MWDA (this work) & 8.176 & 8.238 & 0.0075 & 0.07 \\
\hline & GELA & \multirow{2}{*}{\multicolumn{4}{|c|}{$\begin{array}{l}\text { not attempted } \\
\text { not attempted }\end{array}$}} \\
\hline & MELA & & & & \\
\hline & ELA (Ref. 14) & 11.34 & 11.43 & 0.007 & 0.07 \\
\hline & HO (Ref. 14) & 12.30 & 12.47 & 0.014 & 0.07 \\
\hline & Simulation (Ref. 16) & 5.54 & 5.57 & 0.005 & 0.18 \\
\hline
\end{tabular}

TABLE II. Comparison of Helmholtz-free-energy calculations for the inverse-sixth-power potential: The molecular-dynamics (MD) results are from Ref. 17 and the MWDA and MHNC results are from the present calculation.

\begin{tabular}{ccccc}
\hline \hline$\gamma$ & $\frac{\beta F_{\text {fcc }}}{N}(\mathrm{MD})$ & $\frac{\beta F_{\text {fcc }}}{N}(\mathrm{MWDA})$ & $\frac{\beta F_{\text {liq }}}{N}(\mathrm{MD})$ & $\frac{\beta F_{\text {liq }}}{N}(\mathrm{MHNC})$ \\
\hline 1.897 & 17.058 & & 16.832 & 16.832 \\
2.055 & 19.650 & & 19.510 & 19.496 \\
2.214 & 22.396 & 22.763 & 22.349 & 22.334 \\
2.372 & 25.302 & 25.637 & 25.352 & 25.336 \\
2.530 & 28.373 & 28.667 & & 28.501 \\
2.688 & 31.612 & 31.835 & & 31.833 \\
2.846 & 35.023 & 35.141 & 35.329 \\
\hline \hline
\end{tabular}


ment of the exponential in the Gaussian parametrization [Eq. (29)]. In this respect, the theory fails to correct the deficencies of the earlier theories based on second-order truncations.

Despite their excellent predictions for the hard-sphere freezing transition, the GELA and the MELA both fail to predict freezing for longer-range potentials (here $n$ $=6$ and 12). For both functionals, no minimum was found in either the fcc or bcc solid free energies as a function of $\alpha$ for any value of the solid density. A similar lack of minima for the MELA was found by Barrat and co-workers, ${ }^{41}$ for the truncated repulsive Lennard-Jones, the full Lennard-Jones, and the exponential-6 (similar to Lennard-Jones potential, except with an exponential repulsion) potentials. The reason why these two related theories (MELA and GELA) should fail so completely for soft spheres is, at present, unknown.

\section{RESULTS FOR YUKAWA POTENTIALS}

The Yukawa potential is defined in Eq. (2). This wellknown potential is, among other things, an excellent model for the repulsive screened Coulombic interaction present in monodisperse colloidal systems. ${ }^{24}$ The range of this potential can be tuned by varying the screening parameter $\kappa$. Experimental variation of $\kappa$ is achieved by changing the electrolyte concentration of the fluid in which the colloidal particles are suspended.

Recently, the phase diagram for this interaction has been calculated by Robbins, Kremer, and Grest ${ }^{25}$ using molecular-dynamics computer-simulation techniques. Their results are in good agreement with experiments on colloidal polystyrene spheres (for a description of the experimental findings, see Ref. 25). Note that the phase diagram determined by Robbins, Kremer, and Grest (shown in Fig. 3) is not the usual type in which the boundary lines denote thermodynamic regions of phase coexistence. The phase boundaries on their diagram are the intersection lines of the Helmholtz free-energy surfaces of the various phases, taken at a constant density.

The two parameters that describe a point on this phase diagram are a reduced inverse screening length

$$
\lambda=\kappa a,
$$

and a renormalized reduced temperature

$$
\bar{T}=\frac{T^{*}}{a e^{-\lambda}},
$$

where $a=\rho^{-1 / 3}$ is a typical nearest-neighbor particle separation and $T^{*}=k T / \epsilon$ is the standard reduced temperature. The factor $e^{-\lambda}$ renormalizes the temperature by a typical nearest-neighbor interaction energy.

The form of the Yukawa potential is such that this Helmholtz free-energy phase diagram only depends on $\lambda$ and $\bar{T}$, and not upon the specific constant value of the density at which it is constructed. The construction of the usual type of coexistence diagram would, however, involve the density as an explicit parameter.

The Yukawa phase diagram (Fig. 3) is divided into

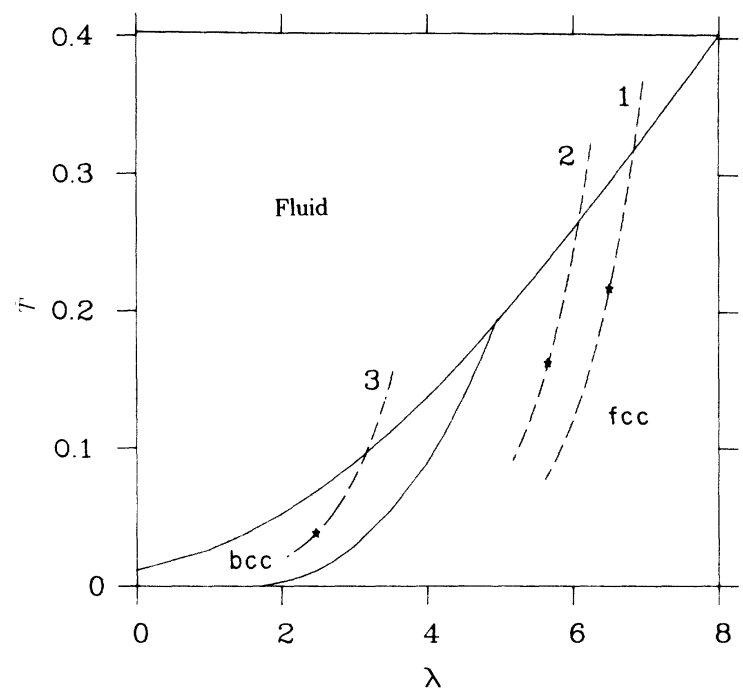

FIG. 3. Phase diagram for the Yukawa fluid. The solid lines are the phase boundaries as calculated by computer simulation (Ref. 25). The stars are points on the fcc-liquid phase boundary as calculated using the MWDA. The dashed lines are lines of constant $\kappa$ and $T^{*}$ (see text).

three regions. In the upper left part of the diagram (low $\lambda$, high $\bar{T}$ ) the fluid is the state with the lowest free energy. The solid phases (fcc and bcc) occupy the lower right "triangle" of the diagram, with the bcc phase restricted to the lower left part of this region (small $\lambda$ and small $\bar{T}$ ). As for the inverse-power systems, the equilibrium solid structure along the liquid-solid boundary changes from fcc to bcc as the effective range of the potential is increased (by decreasing $\lambda$ ). At zero temperature the transition from bcc to fcc occurs at a $\lambda$ of 1.72 . At the freezing point this transition is shifted to about 4.9 .

We have performed freezing calculations using the Denton-Ashcroft MWDA for this potential at $\kappa=1.3$ and at three different reduced temperatures: $T_{1}^{*}=6.5$ $\times 10^{-5}, T_{2}^{*}=1.3 \times 10^{-4}$, and $T_{3}^{*}=1.7 \times 10^{-3}$. These values of the parameters were chosen so that both the fcc $\left(T_{1}^{*}\right.$ and $\left.T_{2}^{*}\right)$ and bcc $\left(T_{3}^{*}\right)$ regions of the phase diagram are covered by the calculation. The liquid structure and free-energy input data were obtained using the same proceedure as described in Sec. III for inverse-power potentials. Given their failure for the inverse-power potentials and the amount of effort required in their implementation, Yukawa freezing calculations using the MELA and the GELA methods were not attempted.

Our results for the fcc-liquid coexistence densities as well as the fractional density change and Lindemann ratio are given in Table III for the three temperatures studied. The corresponding values of $\lambda$ and $\bar{T}$ are also given. As for the inverse-power system, no bcc-liquid transition was found, even for $T_{3}^{*}$, where both the experiments and computer simulation indicate that bcc should be more stable than fcc. For $T_{3}^{*}$, a metastable bcc phase was found, but the Helmholtz free energy of this phase does 
TABLE III. MWDA fluid-solid (fcc) coexistence results for the Yukawa potential with $\kappa=1.3$.

\begin{tabular}{cccc}
\hline \hline & $T_{1}^{*}$ & $T_{2}^{*}$ & $T_{3}^{*}$ \\
& $6.5 \times 10^{-5}$ & $1.3 \times 10^{-4}$ & $1.7 \times 10^{-3}$ \\
\hline$\rho_{l}$ & 0.00795 & 0.01232 & 0.1456 \\
$\rho_{s}$ & 0.00812 & 0.01273 & 0.1459 \\
$\Delta \rho / \rho_{s}$ & 0.021 & 0.032 & 0.002 \\
$L$ & 0.09 & 0.079 & 0.070 \\
$\lambda_{l}$ & 6.514 & 5.629 & 2.471 \\
$\lambda_{s}$ & 6.468 & 5.568 & 2.469 \\
$\tilde{T}_{l}$ & 0.220 & 0.157 & 0.03824 \\
$\bar{T}_{s}$ & 0.208 & 0.146 & 0.03814 \\
\hline \hline
\end{tabular}

not cross either the liquid or fcc free-energy curve for any reasonable density (see Fig. 4)

Because the results in Table III are for liquid-fcc coexistence, they cannot be directly compared to the computersimulation phase diagram (Fig. 3). In order to make this comparison, we have also calculated, for these three temperatures, the densities $\rho_{c}$ at which the fcc and fluid Helmholtz-free-energy curves cross. These densities, as well as the corresponding $\lambda_{c}$ and $\bar{T}_{c}$, are given in Table IV (along with the simulation values). They are also plotted

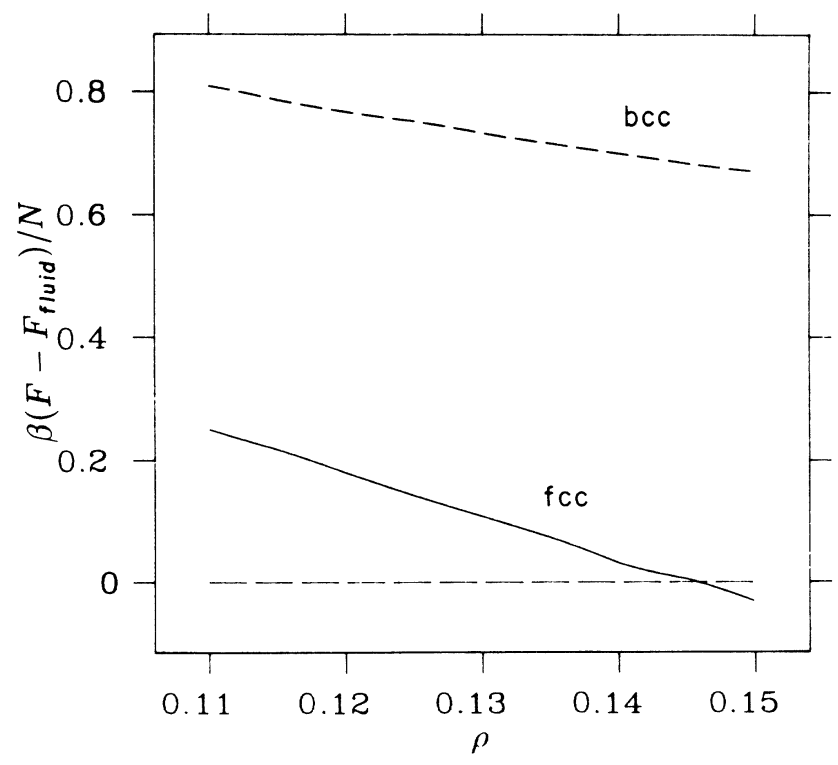

FIG. 4. The dimensionless Helmholtz free energy (relative to the fluid) for fcc and bcc solid structures for a Yukawa fluid with $\kappa=1.3$ and $T^{*}=1.7 \times 10^{-3}$.
TABLE IV. Crossing densities of the Yukawa liquid and fcc solid free-energy curves with $\kappa=1.3$.

\begin{tabular}{ccccc}
\hline \hline \multirow{2}{*}{$T^{*}$} & Method & $\rho_{c}$ & $\lambda_{c}$ & $\bar{T}_{c}$ \\
& & & & \\
\hline \multirow{2}{*}{$6.5 \times 10^{-5}$} & MWDA & 0.00798 & 6.50 & 0.217 \\
& MD & 0.00686 & 6.84 & 0.317 \\
& & & & \\
$1.3 \times 10^{-4}$ & MWDA & 0.0124 & 5.61 & 0.154 \\
& MD & 0.00982 & 6.07 & 0.264 \\
& MWDA & 0.1458 & 2.470 & 0.03818 \\
$1.7 \times 10^{-3}$ & MD(bcc) & 0.0703 & 3.15 & 0.097 \\
& & & & \\
\hline \hline
\end{tabular}

as stars on the phase diagram (Fig. 3). (The dashed lines on the phase diagram represent curves of constant $\kappa$ and $T^{*}$ - the downward direction on these curves represents increasing density.) From Fig. 3, we see that for a given $\lambda$ the value of $\bar{T}$ at which the fcc and fluid free energies are equal is consistently underestimated for all three values of $T^{*}$. From this we can conclude that, for fixed $\kappa$ and $T^{*}$, the melting and freezing densities are overestimated, as in the inverse-power system. Along the liquid-solid line of the phase diagram, the simulations obtain a Lindemann ratio of about $19 \%$. From the values of $L$ given in Table III, we see that, as for the inverse powers, this quantity is about a factor of 2 too small for the MWDA.

\section{DISCUSSION AND CONCLUSIONS}

We have studied the freezing properties of two types of repulsive soft-sphere interactions, inverse-power and Yukawa, using several recently developed weighteddensity-functional theories. For the freezing of these systems into a fcc solid structure, we find that the modified weighted-density approximation of Denton and Ashcroft overestimates the coexistence densities and that the error increases with the range of the potential. This overestimation is, however, significantly less than that of earlier density-functional theories and is primarily due to the fact that the slopes of the liquid and solid free-energy curves are very similar at freezing, which makes the calculated crossing point very sensitive to any error in the free energies. In fact, the calculated fcc free energies for these softer potentials is shown to have about the same relative error as those for hard spheres, even though the hard-sphere transition is predicted with much greater accuracy. No improvement was seen over the earlier theories in the prediction of the Lindemann ratio.

The MWDA fails to predict any fluid to bcc solid transitions, even under conditions at which computer simulations find a thermodynamically stable bcc phase. A metastable bcc phase was found for one of the Yukawa potential calculations, but the free energy was such that it would never (for a reasonable density) be more stable 
than either the liquid or the bcc phase.

For the generalized effective-liquid approximation of Baus and Lutsko and the modified effective-liquid approximation of Baus, no stable or metastable solid phases (fcc or bcc) were found (at least for the inverse-power potentials). Similar failure of the MELA for the LennardJones (both full and truncated) potential has also been recently reported. ${ }^{41}$ The complete failure of these methods is suprising in view of the excellent results they yield for the hard-sphere transition.

Although the MWDA (and therefore the more complicated WDA of Curtin and Ashcroft) gives results for these potentials that are quantitatively better than those of the earlier theories based on a second-order truncation of the functional, the qualitative picture has not changed. The failure to correctly predict freezing into non-close-packed solids remains a major problem with density-functional freezing theories.

Weighted-density-functional theories (of all types) do very well at predicting the hard-sphere freezing transition, even giving a reasonable "metastable" bcc free energy. Why do these methods (especially MELA and GELA) become so much worse as the range of the interaction potential is increased? One possible answer is that the range of the correlations is incorrectly described.

The standard explanation as to why the correlation functions of a solid should be well described by those of a reference liquid with a lower bulk density is that the solid correlations, as a consequence of localization, are weaker than those of a liquid at the same density. ${ }^{8}$ The weighted-density formalism, it is then speculated, provides a good way of determining this "optimum" lower reference density. The problem is that the range of the correlation functions in a liquid is, in general, a relatively strong function of density. Thus it is not necessarily true that the same liquid that gives a good description of the amplitude of the solid correlations simultaneously gives a good estimate of their range-except, perhaps, in the case of hard spheres where the range of the liquid correlations is only very weakly density dependent.

\section{ACKNOWLEDGMENTS}

We would like to thank Professor A.D.J. Haymet and Dr. Steve Rick for the use of their integral-equation program. This material is based upon work supported by the North Atlantic Treaty Organization under a grant given to B.B.L.
${ }^{1}$ A.D.J. Haymet, Annu. Rev. Phys. Chem. 38, 89 (1987).

${ }^{2}$ M. Baus, J. Phys. Condens. Matter 2, 2111 (1990).

${ }^{3}$ A.D.J. Haymet, J. Chem. Phys, 78, 4641 (1983).

${ }^{4}$ B.B. Laird, J.D. McCoy, and A.D.J. Haymet, J. Chem. Phys. 87, 5449 (1987).

${ }^{5}$ B.B. Laird, J.D. McCoy, and A.D.J. Haymet, J. Chem. Phys. 88, 3900 (1988).

${ }^{6}$ G.L. Jones and U. Mohanty, Mol. Phys. 54, 1241 (1985).

${ }^{7}$ M. Baus and J.L. Colot, Mol. Phys. 55, 653 (1985).

${ }^{8}$ W.A. Curtin and N.W. Ashcroft, Phys. Rev. A 32, 2909 (1985).

${ }^{9}$ F. Iglói and J. Hafner, J. Phys. C 19, 5799 (1986).

${ }^{10}$ A.R. Denton and N.W. Ashcroft, Phys. Rev. A 39, 4709 (1989).

${ }^{11}$ J.F. Lutsko and M. Baus, Phys. Rev. Lett. 64, 761 (1990).

${ }^{12}$ C. Marshall, B.B. Laird, and A.D.J. Haymet, Chem. Phys. Lett. 122, 320 (1985).

${ }^{13}$ W.A. Curtin and N.W. Ashcroft, Phys. Rev. Lett. 56, 2775 (1986).

${ }^{14}$ J.L. Barrat, J.P. Hansen, G. Pastore, and E.M. Waisman, J. Chem. Phys. 86, 6360 (1987).

${ }^{15}$ J.P. Hansen, Phys. Rev. A 2, 221 (1970).

${ }^{16}$ W.G. Hoover, M. Ross, K.W. Johnson, D. Henderson, J.A. Barker, and B.C. Brown, J. Chem. Phys. 52, 4931 (1970).

${ }^{17}$ W.G. Hoover, S.G. Gray, and K.W. Johnson, J. Chem. Phys. 55, 1128 (1971).

${ }^{18}$ J.P. Hansen and D. Schiff, Mol. Phys. 25, 1281 (1973).

${ }^{19}$ W.G. Hoover, D.A. Young, and R. Grover, J. Chem. Phys. 56, 2207 (1972).

${ }^{20}$ H. Iyetomi and S. Ichimaru, Phys. Rev. B 38, 6761 (1988).

${ }^{21}$ J.-L. Barrat, J.-P. Hansen, and G. Pastore, Phys. Rev. Lett. 58, 2075 (1987).

${ }^{22}$ M. Baus, J. Phys. Condens. Matter 1, 3131 (1989).

${ }^{23}$ W.A. Curtin and K. Runge, Phys. Rev. A 35, 4755 (1987).

${ }^{24}$ S. Alexander, P.M. Chaikin, P. Grant, G.J. Morales, P.
Pincus, and D. Hone, J. Chem. Phys. 80, 5776 (1984).

${ }^{25}$ M.O. Robbins, K. Kremer, and G.S. Grest, J. Phys. Chem. 88, 3286 (1988).

${ }^{26}$ R. Evans, Adv. Phys. 28, 143 (1979).

${ }^{27}$ T.V. Ramakrishnan and M. Yussouff, Phys. Rev. B 19 2775 (1979).

${ }^{28}$ A.D.J. Haymet and D.W. Oxtoby, J. Chem. Phys. 74, 2559 (1981); A.D.J. Haymet, ibid. 78, 4641 (1983).

${ }^{29}$ P. Tarazona, Mol. Phys. 52, 81 (1984).

${ }^{30}$ R.D. Groot and J.P. van der Eerden, Phys. Rev. A 31, 4155 (1985).

${ }^{31}$ T.F. Meister and D.M. Kroll, Phys. Rev. A 31, 4055 (1985).

${ }^{32}$ The theories of Groot and van der Eerden (Ref. 30) and Meister and Kroll (Ref. 31) differ in that they define the reference density so that the free-energy functional is stationary with respect to variations in $\hat{\rho}$. (They also consider a spatially dependent $\hat{\rho}$, as in the WDA.) Although such an approximation gives resonable results for the density profile of a fluid against a hard wall, it does not lead to a suitable freezing theory (Ref. 33).

${ }^{33}$ D.M. Kroll and B.B. Laird, preceding paper, Phys. Rev. A.

${ }^{34}$ W.J. Hoover and F.M. Ree, J. Chem. Phys. 49, 3609 (1968)

${ }^{35}$ M.O. Robbins, G.S. Grest, and K. Kremer, Phys. Rev. B (to be published).

${ }^{36}$ Y. Rosenfeld and N.W. Ashcroft, Phys. Rev. A 20, 1208 (1979)

${ }^{37}$ F. Lado, S.M. Foiles, and N.W. Ashcroft, Phys. Rev. A 28 , 2374 (1983).

${ }^{38}$ F. Lado, Phys. Lett. A 89, 196 (1982).

${ }^{39}$ Y. Rosenfeld, J. Stat. Phys. 42, 437 (1986).

${ }^{40}$ S. Labík, A. Malijevský, and P. Vońka, Mol. Phys. 56, 709 (1985).

${ }^{41}$ A. de Kuijper, W.L. Vos, J.-L. Barrat, J.-P. Hansen, and J.A. Schouten, J. Chem. Phys. (to be published). 